\title{
Biotransformation of Vincamine using Microbial cultures
}

\author{
Venisetty RK, Keshetty S and Ciddi Veeresham* \\ University College of Pharmaceutical Sciences, Kakatiya University, Warangal AP 506009, India
}

\begin{abstract}
Microbial transformation is a complementary tool in the investigation of drug metabolism. In the present investigation, biotransformation of vincamine was studied using microbial cultures. Bacterial, fungal, and yeast cultures were employed to elucidate the metabolism of vincamine. The results indicate that a number of microorganisms metabolized vincamine to various levels to yield three major metabolites, which were identified by HPLC-DAD and LC-MS-MS analyses. HPLC analysis of the extracts of the cultures showed that 16 out of 39 cultures were able to metabolize vincamine to produce one or more metabolites and the biotransformed products are more polar than the substrate vincamine. Out of 16 cultures maximum biotransformation of vincamine was observed with the cultures of Absidia coerulea MTCC 1335. The major metabolite was hydroxylated metabolite of vincamine, while the other metabolites were produced by dihydroxylation and methyl hydroxylation which are not seen in mammalian metabolism. The results will support the formation of new metabolites and more polar metabolites which have significance in the improvement of pharmacokinetic parameters of vincamine derivatives.
\end{abstract}

Keywords: Biotransformation, Microbial Cell Cultures, Vincamine, Metabolites, HPLC, LC-MS-MS

\section{Introduction}

An important factor in the evaluation of safety and efficacy of any drug is the knowledge of how it is metabolized. Traditionally, drug metabolism studies are conducted on small animal models, perfused organs, cell cultures and enzyme systems [1]. It's a universal fact that microorganisms are sources of many enzymes and they catalyze different types of chemical reactions. Reports [2] indicate that some microorganisms especially fungi are found to possess the cytochrome p450 group of enzymes. The cytochrome p450 enzymes are responsible for a large number of Phase I metabolic pathways. Microbial models may constitute an alternative to the use of animal models, if they can mimic the mammalian metabolism and can give some information about the metabolic fate of the drug. These microbial biotransformation processes are also important to produce newer metabolites which were formed by the introduction of functional groups into unattainable sites of the molecules [3].

Vincamine is an alkaloid obtained from the leaves of Vinca minor L.; or Catharanthus roseus (Apocynaceae). It has been therapeutically used as a vasodilator and antihypertensive agent, particularly in cerebrovascular disorders [4]. Vincamine is used for the prevention and treatment of cerebrovascular insufficiencies and disorders [5]. Vincamine is almost completely metabolized in rats after oral administration. The main urinary metabolites were vincamine conjugates. Radiolabel studies in rats after an oral dose of 10 $\mathrm{mg} / \mathrm{kg}$ body weight demonstrated that vincamine is hydrolysed by the plasma esterases to vincaminic acid which is quickly decarboxylated and oxidized to eburnamenine. Vincamine is also hydroxylated to 6- $\beta$ hydroxyvincamine, which accounts for $40 \%$ of the total urinary and biliary excretion, followed by $6-\alpha-$ hydroxyvincamine (8\%), and conjugated 6-keto-vincamine (10\%) [6].

The aim of present investigation is to study the metabolites of vincamine formed by microorganisms and to compare them with those produced in mammals. To date there is no report on microbial biotransformation of vincamine. Since vincamine is metabolized by CYP group of enzymes by oxidative pathway, microbial cultures particularly fungi may provide an alternative to produce these mammalian metabolites [7]. Fungi serve as a source of CYP and there are chances of producing new metabolites that possess activity which may increases cerebral blood flow or any other interesting activity. Close structural similarity between the agents which enhances the memory power and the possibility of producing new and unusual metabolites with microorganisms prompted us to investigate the microbial metabolism of vincamine. The importance of introduction of polar groups in enhancing the pharmacokinetics and reducing the toxicity of a semisynthetic vincamines such as vinpocetin and ethyl apovincaminate $[8,9]$ are also the reasons for selecting vincamine to study for microbial metabolism. In the present investigation, a screening study was performed by vincamine for its metabolism by 29 fungal, 3 bacterial and 7 yeast cultures. 


\subsection{CHEMICALS}

\section{Materials And Methods}

Vincamine used in the study was in amorphous form, kindly gifted by Covex Pharma, Madrid, Spain. The solvents were purchased from Ranbaxy Fine Chemicals Ltd., New Delhi and all other chemicals of highest available purity were purchased from Hi Media Laboratories Pvt. Ltd., Mumbai. The organisms capable of producing mammalian metabolites were selected and obtained from Microbial Type Culture Collection and Gene Bank (MTCC), Chandigarh, India and National Collection of Industrial Microorganisms (NCIM), NCL, Pune, India.

\subsection{CULTURE PROCEDURE}

Procured cultures were revived, sub cultured and stored in refrigerator at $4{ }^{\circ} \mathrm{C}$. The bacterial, fungal and yeast cultures were maintained on nutrient agar, potato dextrose agar and MGYP (malt extract, glucose, yeast and peptone) agar slants respectively. The media used for biotransformation studies of fungi cultures is Dextrose broth, supplemented with $0.02 \%$ Triton X 100 [10], bacteria is nutrient agar broth and for yeast cultures is MGYP broth. Media was prepared in $50 \mathrm{ml}$ conical flasks with $10 \mathrm{ml}$ media in each flask. The flasks were cotton plugged, covered with aluminum foil and sterilized by autoclaving at $121^{\circ} \mathrm{C}\left(15 \mathrm{lb} \mathrm{in}^{-2}\right)$ for $15 \mathrm{~min}$.

\subsection{BIOTRANSFORMATION}

The second stage cultures $(10 \mathrm{ml}$ culture in $50 \mathrm{ml}$ capacity conical flask) were added with $2 \mathrm{mg}$ each of vincamine (in $100 \mu$ lsolvent (mixture of dichloromethane and methanol in the ratio $1: 3$ )) using a micropipette. Prior to addition, the drug solution was filter sterilized using sterile syringe driven filter unit of $0.45 \mu \mathrm{m}$ (Millipore, MA, USA). The final drug concentration in each flask will be $0.2 \mathrm{~g} \mathrm{l}^{-1}$ culture. The flasks were gently shaken immediately after the addition of drug for its even distribution and minimizing the toxic effects to some cells. The precipitate of vincamine was not visually observed in the culture. Each culture was studied in quadruplicate while running suitable controls. Culture controls consisted of culture blanks in which the organisms were grown under identical conditions but without adding the drug. The culture controls were added with $100 \mu \mathrm{l}$ solvent, the solvent used to dissolve the drug. Drug controls were composed of the sterile medium to which same amount of the drug was added and incubated without microorganisms. The incubation was continued under similar conditions for ten days, the flasks were taken out, extracted and analyzed.

\subsection{EXTRACTION AND SAMPLE PREPARATION OF VINCAMINE AND METABOLITES}

The cultures, after ten days of incubation with vincamine, were taken out and extracted with $3 \times 10 \mathrm{ml}$ of ethyl acetate by vortex mixing for $1 \mathrm{~min}$. The combined organic phase (upper ethyl acetate layers) was evaporated under reduced pressure followed by drying in vacuum oven. The dried samples were reconstituted in $1.5 \mathrm{ml}$ each of HPLC grade methanol by vortex mixing for $1 \mathrm{~min}$. The samples were then taken into micro centrifuge tubes and centrifuged at $12,000 \mathrm{x} \mathrm{g}$ and $20^{\circ} \mathrm{C}$ for $20 \mathrm{~min}$ in micro refrigerated centrifuge. The supernatants were taken out and used for HPLC-DAD and LC-MS-MS analysis.

\subsection{HPLC-DAD ANALYSIS}

The samples prepared after extraction were analyzed by an isocratic HPLC method for presence of metabolites. HPLC analysis was performed on LC-10AT (Shimadzu Corporation, Kyoto, Japan) system by injecting $20 \mu \mathrm{l}$ of sample using Hamilton Rheodyne syringe (Hamilton Bonaduz AG, Switzerland) into syringe loading sample injector (Model 7725i, Rheodyne LP, CA, USA) following the conditions quite close to the method described previously by Dal Bo[11]. The column used was Luna C18, $5 \mu, 250$ x $4.6 \mathrm{~mm}$ i.d. (Phenomenex, USA). The mobile phase consisted of a mixture of acetonitrile-methanol-buffer (10mM ammonium acetate $\mathrm{pH}$ adjusted to 7.3 with triethylamine) in 20:30:50 ratio. The mobile phase was degassed using ultrasonic bath (Sonorex, Bandelin Electronic, Germany). The analysis was performed isocratically at a flow rate of $1 \mathrm{ml} / \mathrm{min}$ at ambient temperature. Drug and its metabolites were detected using diode array detector (Shimadzu SPD M10Avp model, Shimadzu Corporation, Kyoto, Japan) at a wavelength of $270 \mathrm{~nm}$. The data analysis was performed by Class M10 software (Shimadzu Corporation, Kyoto, Japan). The UV absorption spectrum of the metabolites was compared with that of vincamine. The metabolites were quantified based on the peak areas and expressed as percentages of metabolites formed. The calculations were performed with respect to the total area of drug and metabolites together taken as $100 \%$.

\subsection{LC-MS-MS ANALYSIS}

The $\mathrm{m} / \mathrm{z}$ values of drug and metabolites and their fragmentation ions were recorded by LC-MS-MS using Perkin Elmer Sciex API mass spectrometer set in positive mode. The API-4000 LC-MS/MS was operated under the multiple reaction-monitoring mode (MRM) using the electrospray ionization technique. The separation was performed on X-Terra MS C-18 column $(4.6 \mathrm{~mm}$ x $50 \mathrm{~mm}, 5 \mu)$. The mobile phase consisted of a 
mixture of acetonitrile: methanol: buffer (10mM ammonium acetate $\mathrm{pH}$ adjusted to 7.3 with triethylamine) in 20:30:50 ratio, was isocratically pumped at a flow rate of $1 \mathrm{ml} / \mathrm{min}$. 50 $\mu 1$ of the sample was injected using auto sampler device. The vaporizer temperature and the discharge current were set at $300^{\circ} \mathrm{C}$ and $10 \mu \mathrm{A}$ respectively. The fragments were scanned in the $\mathrm{m} / \mathrm{z}$ range of 50 to 500 and the data were processed with Sciex Analyst software. The metabolites were identified basing on pattern of UV spectra in HPLC-DAD and $\mathrm{m} / \mathrm{z}$ values of the fragmentation products obtained in LC-MS-MS analysis.

\section{Results}

There was no significant change in the medium $\mathrm{pH}$ after the addition of the drug. In all the three drug controls (i.e., drug in three different media) studied, none has shown any additional peaks in HPLC analysis indicating that the substrate vincamine was stable in the media. The $\%$ recoveries of vincamine from these media were $>92.3 \%$.

HPLC analysis of the extracts of the cultures showed that 16 out of 39 cultures were able to metabolize vincamine to produce one or more metabolites evidenced from the absence of these new peaks in drug control as well as culture controls. Out of 16 microbial cultures the maximum biotransformation of Vincamine was observed with the cultures of Absidia coerulea MTCC 1335. Representative HPLC chromatograms were shown in "Fig. 1 and Fig. 2".

Vincamine was eluted at $21 \mathrm{~min}$ and the metabolites produced were designated as M1, M2 and M3 were eluted at $4.9 \mathrm{~min}, 7.8 \mathrm{~min}$ and $8.4 \mathrm{~min}$ respectively. Control samples with and without vincamine showed no coincidental peaks eluting with identified metabolites. The results pertaining to the metabolite production was shown in Table 1. All the new peaks had shown nearly similar UV spectral pattern from 190 to $370 \mathrm{~nm}$

A large variation was observed in the quantitative metabolite production among various cultures. M3 was the metabolite found in most of the cultures. Absidia coerulea MTCC 1335 produced $31.76 \%$ of this metabolite whereas Rhizopus stolonifer NCIM 880 produced only $7.93 \%$ of this metabolite.

The metabolites M1, M2 and M3 had shown the protonated molecular ions at $\mathrm{m} / \mathrm{z}$ values 387.2, 401.2 and 371.3 respectively. The parent drug Vincamine has shown protonated molecular ion at $\mathrm{m} / \mathrm{z} 355.3$. Table 2 represents the retention times, $\mathrm{m} / \mathrm{z}$ values of parent ions $(\mathrm{M}+\mathrm{H})^{+}$and predicted molecular formulae and metabolic reactions for various metabolites. The ionization spectrum of the $\mathrm{m} / \mathrm{z} 355.3$ pertaining to vincamine showed product ions at $\mathrm{m} / \mathrm{z} 337.3,308.4,294.2,280.3$ and 236.3. Sequential losses of $\mathrm{H}_{2} \mathrm{O}(18), \mathrm{C}_{2} \mathrm{H}_{5}(29)$, $\mathrm{CH}_{2}(14), \mathrm{CH}_{2}$ (14) and $\mathrm{CO}_{2}$ (44) was observed.

HPLC retention time of M1 is $4.91 \mathrm{~min}$. Fragmentation pattern of M1 by LC-MS-MS was shown in Figure 3. M1 gave the protonated molecular ion at $\mathrm{m} / \mathrm{z} 387.2$ that is 32 higher than the parent drug giving a clue that hydroxylation took place twice. The ionization spectrum of the $\mathrm{m} / \mathrm{z} 387$ showed product ions at $\mathrm{m} / \mathrm{z} 369.2$, 353.2, 336.2, 308.4, 294.2, 280.3 and 236.1. Sequential losses of $\mathrm{H}_{2} \mathrm{O}$ (18), O (16), OH (17), $\mathrm{C}_{2} \mathrm{H}_{4}(28), \mathrm{CH}_{2}$ (14), $\mathrm{CH}_{2}$ (14) and $\mathrm{CO}_{2}$ (44).

HPLC retention time of M2 is 7.36min. Fragmentation pattern of M2 by LC-MS-MS was shown in Figure 4. M2 gave the protonated molecular ion at $\mathrm{m} / \mathrm{z} 401.2$ that is 46 higher than the parent drug. The ionization spectrum of the $\mathrm{m} / \mathrm{z} 401$ showed product ions at $\mathrm{m} / \mathrm{z} 383.3,366.3,338.3,324.3,308.3,294.8,280.4$ and 236.1. Sequential losses of $\mathrm{H}_{2} \mathrm{O}$ (18), $\mathrm{OH}(17), \mathrm{C}_{2} \mathrm{H}_{4}(28), \mathrm{CH}_{2}(14), \mathrm{O}(16), \mathrm{CH}_{2}(14), \mathrm{CH}_{2}(14)$ and $\mathrm{CO}_{2}$ (44).

HPLC retention time of M3 is $8.44 \mathrm{~min}$. Fragmentation pattern of M3 by LC-MS-MS was shown in Figure 5. M3 gave the protonated molecular ion at $\mathrm{m} / \mathrm{z} 371.3$ which is 16 units higher than vincamine. The ionization spectrum of the $\mathrm{m} / \mathrm{z} 371.3$ showed product ions at $\mathrm{m} / \mathrm{z} 353.3,336.4,308.4,294.2,280.3$ and 236.1 . Sequential loss of $\mathrm{H}_{2} \mathrm{O}$ (18), $\mathrm{OH}(17), \mathrm{C}_{2} \mathrm{H}_{4}$ (28), $\mathrm{CH}_{2}$ (14), $\mathrm{CH}_{2}$ (14) and $\mathrm{CO}_{2}$ (44). Figure 6 represents the proposed biotransformation pathway of vincamine by Absidia coerulae MTCC 1335.

\section{Discussion}

The effect of drug addition on the $\mathrm{pH}$ of the media was studied since $\mathrm{pH}$ is an important factor in the growth and physiology of the microorganism and also in the biotransformation. All the bacterial and yeast cultures produced fine suspensions in liquid media, but the fungi studied produced matt forming cultures. To avoid matt formation Triton X 100 was added to the medium [10]. This also improved the permeability and thus increased the uptake vincamine.

During the first stage, all the bacterial, yeast and fungal cultures produced fine suspensions in liquid media and attained good growth within 48hours. Second stage culture was used for the biotransformation study. Many organisms produced the same range of metabolites, but in different relative amounts. The production of metabolites by microbial cultures may be due to the presence of enzymes expressed naturally or induced by the drug or media component(s). All the metabolites produced were eluted before the drug indicating that they are more polar than vincamine. The relative polarity of the metabolites also helped in their characterization. 
In HPLC method development, the retention time of the drug was set at around 21 min so that the metabolites, being more polar in general, will elute before the drug. The metabolite peaks were identified in HPLC basing on the similarity in UV spectra in the diode array detector. All these new peaks had shown similar UV spectral pattern (from 190 to $370 \mathrm{~nm}$ ) with that of vincamine indicating that the drug has undergone only minor structural changes in the process of metabolism by microorganisms. This also indicates that only some groups have been added from the structure and this observation reveals that there was no loss of aromaticity, ring fission or ring fusion since any of these changes would be expected to lead to a high alteration of the metabolites' UV spectra. The UV $\lambda_{\max }$ of the metabolites were within a narrow range with that of vincamine which indicates that their extinction coefficient at $270 \mathrm{~nm}$ was not significantly different from that of the parent drug. Cha reported that the fungi Cunninghamella elegans was able to N-demethylate malachite green, where the metabolites showed a slight decrease (618 to $608 \mathrm{~nm}$ ) in absorption maxima (visible $\lambda_{\max }$ ) than the substrate malachite green [12].

The metabolites were quantified basing on their areas and expressed as percentages of metabolites formed with respect to the total area of drug and metabolites together taken as $100 \%$. Zhang in their study on the fungal metabolism of cyclobenzaprine, quantified the metabolites basing on their areas by expressing as a percent of the drug added originally in the drug control [13]. Hansen Jr. calculated the single metabolite formation based on the initial dose of triprolidine added after 10 days of incubation, and found that the drug and its metabolites were accounted for 23 and 55\% respectively [14]. They assumed that the remaining $22 \%$ of the initial dose (may contain both drug and its metabolite) might be adsorbed by the fungal cells.

The low production of M3 in Rhizopus stolonifer NCIM 880 may be attributed to many reasons such as expression of low levels of enzymes required for this reaction, substrate/product inhibited biotransformation, non-optimal conditions (media and incubation) for biotransformation, etc.,

The structure elucidation of the metabolites was carried out from the fragmentation results obtained in LC-MS-MS analysis. The structures of the metabolites were proposed from the $\mathrm{m} / \mathrm{z}$ values of the fragmentation ions and HPLC retention times.

Basing on the mass fragmentation and HPLC retention time, M3 was assumed as a hydroxyl metabolite of vincamine, where the hydroxylation took place on the terminal methyl of ethyl side chain of vincamine. This metabolite was not reported in mammals so far. Hansen Jr. reported the formation of hydroxymethyl metabolite of triprolidine using the fungi Cunninghamella elegans, where the $-\mathrm{CH}_{3}$ group present on the benzene ring was hydroxylated to $-\mathrm{CH}_{2} \mathrm{OH}$ and this reaction was mediated by CYP [14]. Cheng found that Streptomyces griseus was able to perform hydroxylation of methyl group at C-30 position of quinovic acid 3-O- $\beta$-Dquinovopyranoside and reported that this is a new oxidized metabolite which was difficult to achieve by chemical means [15]. In the present study, 12 cultures have produced this metabolite. Since this metabolite formation occurs by direct oxidation of methyl group on the vincamine molecule, no specific pathway is involved in this reaction. This suggests that M3 might be hydroxylation product of vincamine.

Basing on the mass fragmentation and HPLC retention time, M1 was assumed as a dihydroxyl metabolite of vincamine where the hydroxylations took place on the terminal methyl group of ethyl side chain and on the eburnamenine ring of vincamine. The favorable position for the hydroxylation on eburnamenine ring is either $6 \alpha$ or $6 \beta$. Formation of this metabolite may take place from M3, which underwent hydroxylation again to produce the dihydroxylated metabolite M1. This metabolite was also not reported in mammalian metabolism. Vigne reported this type of double regiospecific hydroxylation of exo-3-pinanyl-N-phenyl carbamate and exo3-pinanyl-N,N-methylphenyl carbamate where one hydroxylation occurs on terminal methyl group of the pinanyl moiety and a second hydroxylation takes place on the aromatic ring [16]. The study also reported that, when a compound possess an aromatic ring and an alkyl group, the hydroxylation first occurs on the alkyl group and that the alcohol thus formed serves as a substrate to undergo second hydroxylation on the aromatic ring leading to the dihydroxylated product. Hence, in the present study, it can be assumed that the formation of M1 might take place from M3. This suggests that M1 might be dihydroxylated product of vincamine.

Basing on the mass fragmentation and HPLC retention time, M2 was assumed as a methoxy and hydroxyl metabolite of vincamine where the hydroxylation took place on the terminal methyl group of ethyl side chain and methylation on the hydroxyl (M1) of eburnamenine ring of vincamine (O- methylation) . Hosny reported the methylation of 3' and 4' hydroxyl of fistein, 3' O-methylation of catechin and O-methylation of quercitin by Streptomyces gresius [17]. Similarly Hosny and Rosazza reported O-methylation of genistein by Streptomyces [18]. Hence, it is assumed that the formation of this metabolite may take place from M3, which underwent hydroxylation again to produce the dihydroxylated metabolite M1 and the $2^{\text {nd }}$ hydroxyl group i.e., the hydroxylation on eburnamenine ring of vincamine underwent methylation to form M2. This metabolite was also not reported in mammalian metabolism.

Some of the cultures employed in the present study did not produce any metabolites for vincamine. This may be attributed to the absence of substrate specific enzymes required for biotransformation. It may also 
be due to the substrate not reaching the site of biotransformation, i.e., lack of membrane transport processes involved to get the substrate into the site where the enzymes are known to be located.

\section{Conclusion}

At the outset, three microbial metabolites were identified from the microbial cultures screened. All the three metabolites were not reported in mammals. It was suggested that the metabolite M3 (Hydroxy metabolite) is formed first which underwent further hydroxylation to form more polar metabolite M1 (dihydroxylated metabolite). The third metabolite M2 is formed by the methylation of the second hydroxy group and was found to be less polar when compared to M1 but it is a little more polar when compared to M3. These observations were confirmed by the retention times of the metabolites by RP-HPLC and LC-MS-MS analysis in which M1 being more polar of the three metabolites was eluted first at 4.9 min., M2 being intermediately polar was eluted at $7.8 \mathrm{~min}$. and $\mathrm{M} 3$ being the least polar of the three metabolites was eluted at $8.4 \mathrm{~min}$.

\section{Acknowledgements}

Venisetty RK is thankful to the AICTE for financial support and the authors are thankful to Dr. Ramesh Mullangi, Mr. Vittal Sivva and Mr. Raja Reddy Kallem, Discovery research, Dr.Reddy's laboratories, Hyderabad for LC-MS-MS analysis.

\section{References}

[1] A.M. Clark and C.D. Hufford, Use of microorganisms for the study of drug metabolism, an update. Medicinal Research Reviews. 11(5), 1991, 473-501.

[2] J.P. Ferris, L.H. MacDonald, M.A. Patrie and M.A. Martin, Aryl hydrocarbon hydroxylase activity in the fungus Cunninghamella bainieri: Evidence for the presence of cytochrome P-450. Archives of Biochemistry and Biophysics. 175(2), 1976, $443-452$.

[3] R.K. Venisetty and V. Ciddi, (2003) Application of microbial biotransformation for the new drug discovery using natural drugs as substrates. Current Pharmaceutical Biotechnology. 4(3), 2003, 153-167

[4] T. Fleming ed. PDR for Herbal Medicines. (New Jersey: Medical Economics Company. 2000) Pp. 584-585

[5] P. Cook and I. James, Cerebral vasodilators. The New England Journal of Medicine. 305, 1981, $1560-1564$.

[6] V. Vigano, S. Paracchini, G. Piacenza and E. Pesce, Metabolism of vincamine in the rat. Il Farmaco; edizione scientifica. 33(8), 1978, 343-350.

[7] R.K. Venisetty, S. Keshetty and V. Ciddi, Biotransformation of silibinin (silybin) using fungal organisms. Indian Journal of Pharmacy Education and Research. 45(4), 2011, 384-391.

[8] E. Cholnoky and L.L. Doemoek, Summary of safety tests of ethyl apovincaminate. Arzneimittelforschung 26, 1976, $1938-1944$.

[9] K.L. Keim and P.C. Hall, General neuropharmacology of vinpocetine: putative cerebral activator. Drug Development Research. 11(2), 1987, 107-115.

[10] R.K. Venisetty, S. Keshetty and V. Ciddi, Optimization of surfactants and polymers to alter the morphology of matt forming fungal cultures, an improved way fungal drug metabolism studies. Poster presented in $64^{\text {th }}$ International Pharmaceutical Federation Congress in September 2004 held at New Orleans, USA.

[11] L. Dal Bo, G. Ceriani and G. Broccali, Determination of vincamine in human plasma by high-performance liquidchromatography with ultraviolet detection. Journal of Chromatography. 573(1), 1992, 158-162

[12] C.J. Cha, D.R. Doerge and C.E. Cerniglia,Biotransformation of malachite green by the fungus Cunninghamella elegans. Applied and Environmental Microbiology. 67(9), 2001, 4358-4360.

[13] D. Zhang, F.E. Evans, J.P. Freeman, Y. Yang, J. Deck and C.E. Cerniglia, Formation of mammalian metabolites of cyclobenzaprine by the fungus Cunninghamella elegans. Chemico-Biological Interactions. 102(2), 1996, 79-92.

[14] E.B. Hansen Jr., R.H. Heflich, W.A. Korfmacher, D.W. Miller and C.E. Cerniglia, Microbial transformation of the antihistamine drug triprolidine hydrochloride. Journal of Pharmaceutical Sciences. 77(3), 1988, 259-264.

[15] Z.H. Cheng, B.Y. Yu, Y.L. Guo, and S.X. Qiu, Microbial hydroxylation of quinovic acid glycoside by Streptomyces griseus ATTC 13273. Chinese Journal of Chemistry. 24, 2006, 95-98.

[16] B. Vigne, A. Archelas and R. Furstoss, Microbial transformations 18. Regiospecific para-hydroxylation of aromatic carbamate mediated by the fungus Beauveria sulfurescens. Tetrahedron. 47(8), 1991, 1447-1458.

[17] M. Hosny, K. Dhar and J.P.N. Rosazza, Hydroxylation and methylations of quercetin, fisetin and catechin by Streptomyces griseus. Journal of Natural Products. 64(4), 2001, 462-465.

[18] M. Hosny and J.P. Rosazza, Microbial Hydroxylation and Methylation of Genistein by Streptomycetes. Journal of Natural Products. 62(12), 1999, 1609-1612.

\section{FIGURE LEGENDS}

Figure 1. Representative HPLC chromatogram of vincamine in media (Control 1)

Figure 2. Representative HPLC chromatogram of vincamine biotransformation by Absidia coerulea MTCC 1335

Figure 3. Fragmentation pattern of metabolite M1 by LC-MS-MS

Figure 4. Fragmentation pattern of metabolite M2 by LC-MS-MS

Figure 5. Fragmentation pattern of metabolite M3 by LC-MS-MS

Figure 6. Proposed biotransformation pathway of vincamine by Absidia coerulae MTCC 1335. 
Table 1. Metabolites of vincamine produced by microbial cultures

\begin{tabular}{|c|c|c|c|c|}
\hline Culture & Class & M1 (4.9min) & M2 (7.8min) & M3 (8.4min) \\
\hline Absidia coerulea MTCC 1335 & Fungi & 38.21 & 13.57 & 31.76 \\
\hline Absidia glauca MTCC 982 & Fungi & 12.17 & - & 28.13 \\
\hline Aspergillus flavipes NCIM 1209 & Fungi & - & - & - \\
\hline Aspergillus flavus NCIM 554 & Fungi & - & - & - \\
\hline Aspergillus flavus NCIM 557 & Fungi & - & - & - \\
\hline Aspergillus niger NCIM 1006 & Fungi & - & - & - \\
\hline Aspergillus niger NCIM 589 & Fungi & - & - & - \\
\hline Aspergillus niger NCIM 620 & Fungi & - & - & - \\
\hline Aspergillus ochraceous NCIM 1140 & Fungi & - & - & - \\
\hline Aspergillus parasiticus NCIM 898 & Fungi & - & - & - \\
\hline Beauvaria bassiana NCIM 1216 & Fungi & - & - & - \\
\hline $\begin{array}{l}\text { Cunninghamella blakesleana NCIM } \\
687\end{array}$ & Fungi & - & - & 22.10 \\
\hline $\begin{array}{l}\text { Cunninghamella blakesleana NCIM } \\
688\end{array}$ & Fungi & - & - & 12.29 \\
\hline $\begin{array}{l}\text { Cunninghamella echinulata NCIM } \\
691\end{array}$ & Fungi & - & - & 16.17 \\
\hline $\begin{array}{l}\text { Cunninghamella echinulata NCIM } \\
693\end{array}$ & Fungi & - & - & 18.32 \\
\hline Cunninghamella elegans NCIM 689 & Fungi & - & - & - \\
\hline Cunninghamella elegans NCIM 690 & Fungi & - & - & 14.89 \\
\hline Cunninghamella sp. NCIM 1184 & Fungi & - & - & 12.16 \\
\hline Curvularia lunata NCIM 716 & Fungi & - & - & - \\
\hline Fusarium oxysporum NCIM 1008 & Fungi & - & - & - \\
\hline Mucor plumbeus NCIM 984 & Fungi & 10.6 & - & 11.3 \\
\hline Mucor rouxii MTCC 386 & Fungi & 7.5 & - & 15.8 \\
\hline $\begin{array}{l}\text { Pencillium brevicompactum MTCC } \\
549\end{array}$ & Fungi & - & - & - \\
\hline Pencillium chrysogenum NCIM 733 & Fungi & - & - & - \\
\hline Pencillium chrysogenum NCIM 738 & Fungi & - & - & - \\
\hline Rhizopus arrhizus NCIM 997 & Fungi & 8.52 & - & 9.14 \\
\hline Rhizopus stolonifer NCIM 880 & Fungi & 3.54 & - & 7.93 \\
\hline Thamnostylum piriforme NCIM 974 & Fungi & - & - & - \\
\hline Trichothecium roseum NCIM 1147 & Fungi & - & - & 18.15 \\
\hline Bacillus subtilis MTCC 619 & Bacteria & - & - & - \\
\hline Escherichia coli MTCC 118 & Bacteria & - & - & - \\
\hline Pseudomonas putida NCIM 2782 & Bacteria & - & - & - \\
\hline Rhodotorula rubra NCIM 3172 & Yeast & - & - & - \\
\hline $\begin{array}{l}\text { Saccharomyces cerevesiae NCIM } \\
3090\end{array}$ & Yeast & - & - & - \\
\hline Streptomyces griseus NCIM 2622 & Yeast & 12.39 & 9.46 & 16.15 \\
\hline Streptomyces griseus NCIM 2623 & Yeast & 11.15 & 8.18 & 13.35 \\
\hline $\begin{array}{l}\text { Streptomyces lavendulae NCIM } \\
2827\end{array}$ & Yeast & - & - & - \\
\hline Streptomyces rimosus NCIM 2213 & Yeast & 12.68 & - & 21.32 \\
\hline Streptomyces sp. NCIM 2214 & Yeast & - & - & - \\
\hline
\end{tabular}

Note: Values indicate the percentage of metabolites' area in HPLC analysis. The value is the percentage of a metabolite with respect to the total area of drug and all the metabolites formed. 
Table 2. Retention times, $\mathrm{m} / \mathrm{z}$ values and predicted reactions involved in the biotransformation of vincamine by microbial cultures.

\begin{tabular}{|l|l|l|l|l|}
\hline Metabolite & Rt & $\mathbf{m} / \mathbf{z}$ value & Predicted reaction & Predicted Molecular formulae \\
\hline Vincamine & 21.2 & 355.3 & - & $\mathrm{C}_{21} \mathrm{H}_{27} \mathrm{~N}_{2} \mathrm{O}_{3}$ \\
\hline M1 & 4.9 & 387.2 & Dihydroxylation & $\mathrm{C}_{21} \mathrm{H}_{27} \mathrm{~N}_{2} \mathrm{O}_{5}$ \\
\hline M2 & 7.8 & 401.2 & $\begin{array}{l}\text { Hydroxylation } \\
\text { Methoxylation }\end{array}$ & $\mathrm{C}_{22} \mathrm{H}_{29} \mathrm{~N}_{2} \mathrm{O}_{5}$ \\
\hline M3 & 8.4 & 371.3 & Hydroxylation & $\mathrm{C}_{21} \mathrm{H}_{27} \mathrm{~N}_{2} \mathrm{O}_{4}$ \\
\hline
\end{tabular}

Figure 1. Representative HPLC chromatogram of vincamine in media (Control 1)

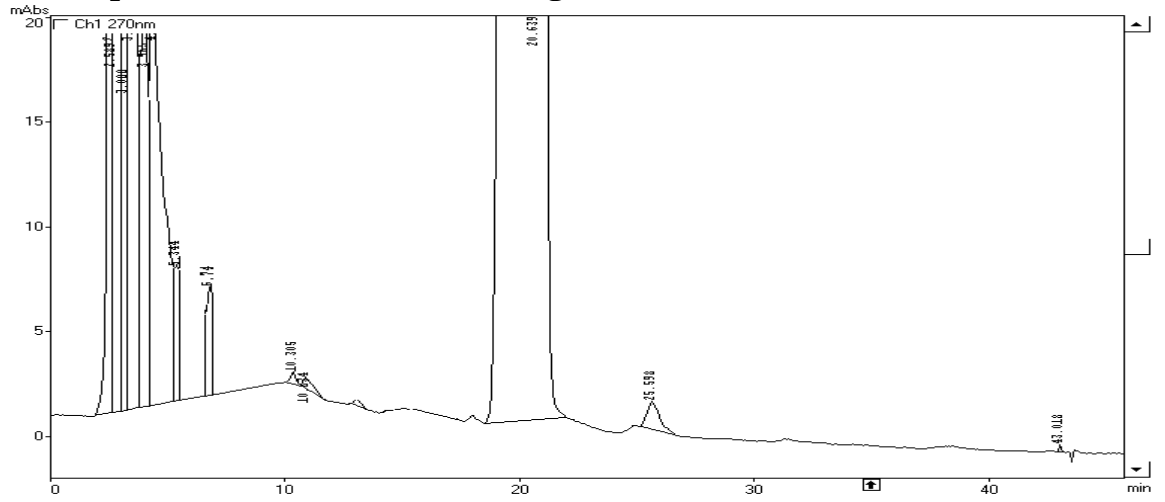

Figure 2. Representative HPLC chromatogram of vincamine biotransformation by Absidia coerulea MTCC 1335

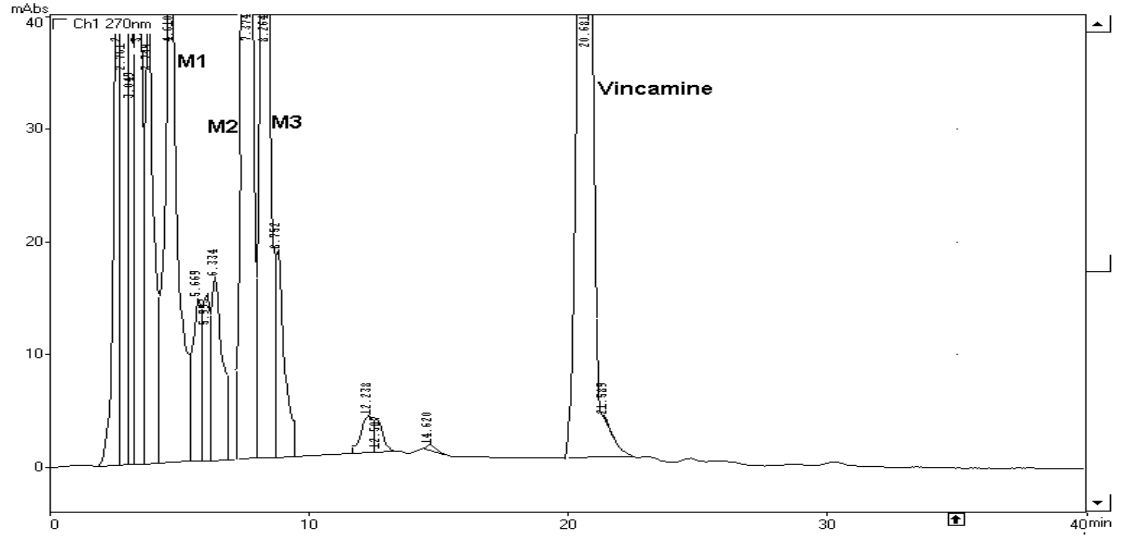

Figure 3. Fragmentation pattern of metabolite M1 by LC-MS-MS

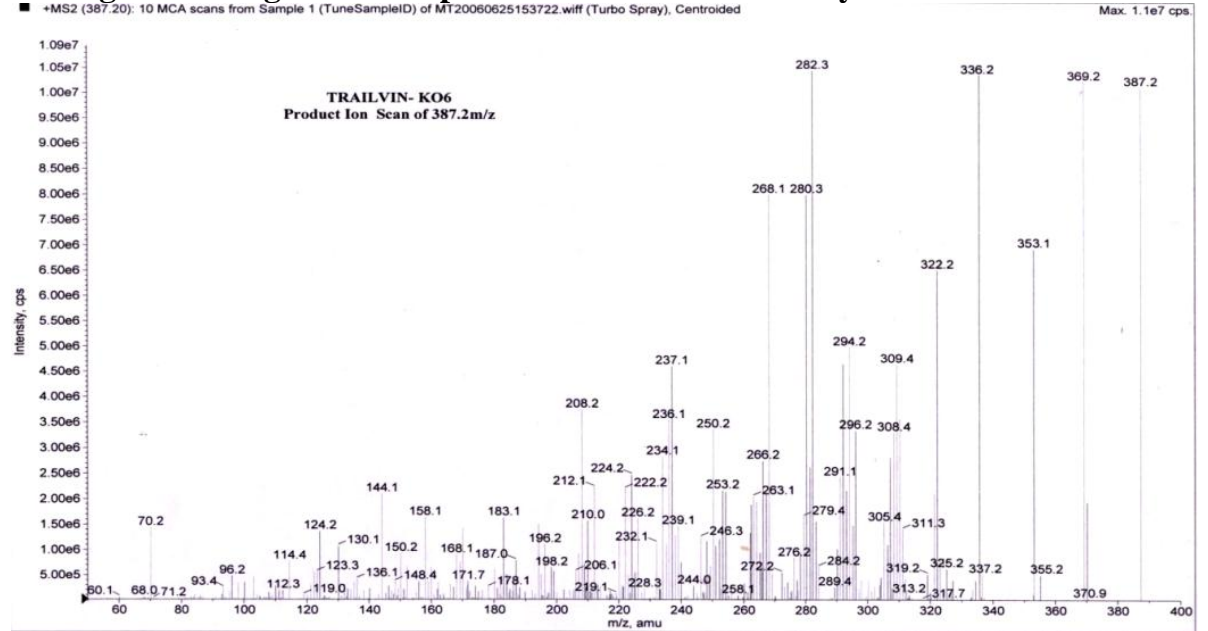


Figure 4. Fragmentation pattern of metabolite M2 by LC-MS-MS
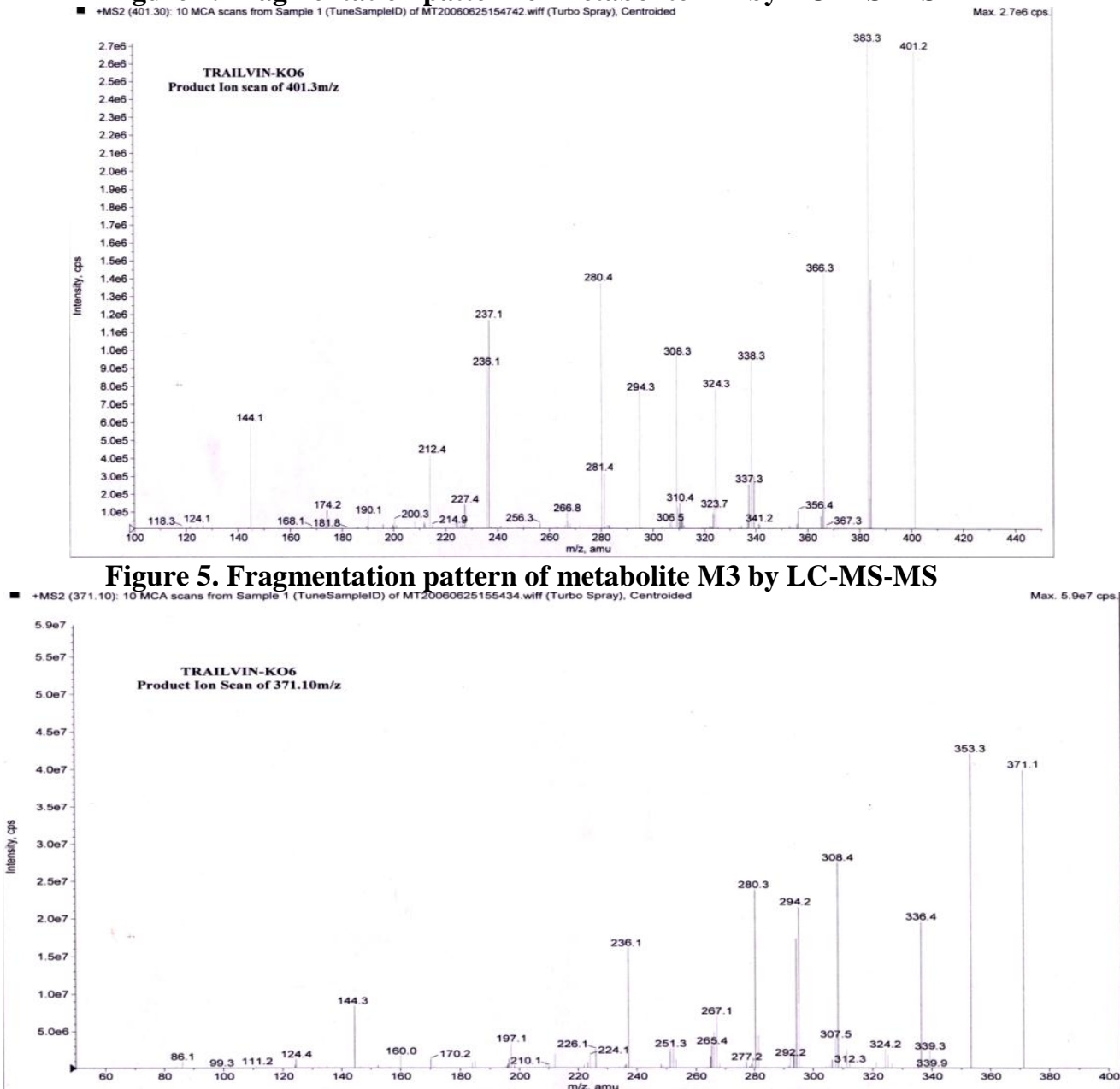

Figure 6. Proposed biotransformation pathway of vincamine by Absidia coerulae MTCC 1335.<smiles>CCC12CCCN3CCc4c(n(c5ccccc45)[C@H]1O)[C@@]32CC(C)=O</smiles>

hydroxylation

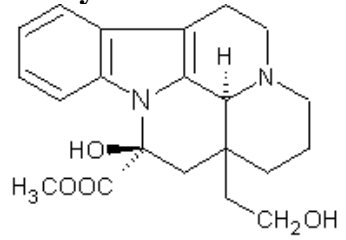

Vincamine, $\mathrm{m} / \mathrm{z} 355.3$

Metabolite M3, $\mathrm{m} / \mathrm{z} 371.1$<smiles></smiles>

\section{O-methylation}

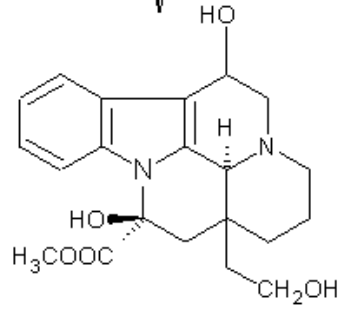

